\title{
AVIFAUNA EM FRAGMENTOS DA MATA ATLÂNTICA
}

\author{
AVIFAUNA IN ATLANTIC FOREST FRAGMENTS
}

\author{
Fábio Rossano Dário ${ }^{1}$ Maria Cristina Veiga De Vincenzo ${ }^{2}$ \\ Álvaro Fernando de Almeida ${ }^{3}$
}

\section{RESUMO}

O estudo foi realizado em área localizada na Serra do Mar, no domínio da Mata Atlântica, na formação florestal denominada Floresta Ombrófila Densa Atlântica, no Município de Paraibuna, Estado de São Paulo, Brasil (localizado entre

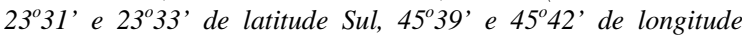
Oeste, e entre 944 a 1,136m de altitude). Foram estudados três fragmentos de vegetação natural de 47, 26 e 4 hectares, e uma área de plantio de eucaliptos adjacente, sendo a vegetação dos fragmentos composta por mata ciliar em estágio médio de regeneração secundária. $O$ método utilizado para o registro da avifauna foi o de observações por pontos fixos. O principal objetivo foi analisar os grupos de aves afetados pela fragmentação florestal e avaliar os impactos causados pela fragmentação e isolamento destas áreas. Considerando-se o método utilizado e todas as áreas estudadas, foi possível registrar 113 espécies de aves, distribuídas em 27 famílias e 13 ordens, sendo as principais guildas a insetivora, onivora e granívora, todas ocupando o sub-bosque. A composição de espécies foi afetada pela fragmentação e redução da área de vegetação natural. A quantidade e diversidade de aves apresentou relação direta com o tamanho dos fragmentos e estrutura da vegetação, $e$ inversa com o grau de isolamento.

Palavras-chave: avifauna, fragmento, Mata Atlântica, subbosque.

\section{SUMMARY}

The study was carried out in an area located at "Serra do Mar", belonging to "Mata Atlântica" formation, also known as Atlantic Rain Forest, near the town of Paraibuna, State

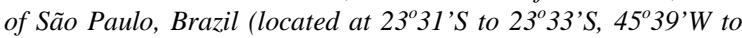
$45^{\circ} 42^{\prime} \mathrm{W}$ and 944 to $1.136 \mathrm{~m}$ altitude). Three fragments of natural vegetation (sizes of 47, 26 and 4 hectares) and an adjacent area of eucalypts plantation were utilized for the surveys. In all situations the understorey was quite prominent and the vegetation of the gallery forest fragments was in a medium stage of secondary regeneration. The method used to the register the avifauna specimens was the technique of observations per fixed points. The main objective of the study was to analyze the groups OF birds that were affected by the forest fragmentation and, additionally, to evaluate the impacts caused by the fragmentation and the isolation degree of these areas. Taking into account all studied areas, it was possible to register a total of 113 species of birds, distributed over a range of 27 families and 13 orders. The main species were cHaracterized as insect-eating, omnivorous and grain-eating, all of them occupying the understorey level. The species composition was affected by the fragmentation and also by the reduction of the natural vegetation. The quantity and diversity of birds showed a direct relationship with the size of the fragments, and structure of the vegetation and an inverse relationship with the isolation degree.

Key words: bird, fragment, Atlantic Forest, understorey.

\section{INTRODUÇÃO}

A Mata Atlântica está situada no Estado de São Paulo, nas encostas da Serra do Mar, com penetrações mais para o interior, particularmente no sul do Estado (LEITÃO FILHO, 1982). Tem seu posicionamento ligado ao relevo, umidade e precipitação atmosférica, o que propicia uma formação de árvores altas, com sub-bosque sombrio

\footnotetext{
${ }^{1}$ Engenheiro Florestal, MSc., Pesquisador, Facoltà di Agraria, Università degli studi di Pisa, Itália.

${ }^{2}$ Engenheiro Agrônomo, MSc., Pesquisador, Dipartamento di Chimica e Biotecnologie Agrarie, Facoltà di Agraria, Universitá degli Studi di Pisa, Itália. Via del Borghetto, 80, 56124, Pisa, Itália. E-mail: crisvicenzo@ hotmail.com. Autor para correspondência.

${ }^{3}$ Biólogo, professor, Doutor, Departamento de Ciências florestais, Escola superior de Agricultura Luiz de Queiróz, Universidade de São Paulo, SP.
} 
e úmido, onde vegetam inúmeras ervas e epífitas (JOLY et al., 1991).

$\mathrm{O}$ acelerado processo de fragmentação da Mata Atlântica tornou este bioma uma das áreas de maior prioridade para a conservação biológica em todo o mundo, sendo que grande parte dos fragmentos sofreu algum tipo de perturbação antrópica (DÁRIO \& ALMEIDA, 2000).

O número de espécies que um fragmento florestal pode suportar e as suas respectivas taxas de extinção dependem do seu tamanho, da distância de uma fonte de povoamento e da estrutura do habitat (MacARTHUR \& WHITMORE, 1979).

A diminuição de uma área de floresta natural pode levar à diminuição exponencial do número de espécies e afetar a dinâmica de populações de plantas e animais existentes, podendo comprometer a regeneração natural e, conseqüentemente, a sustentação destas florestas (HARRIS, 1984).

A fragmentação torna-se um problema quando não há migração e a qualidade do habitat é muito pobre ou a área é muita pequena para sustentar populações viáveis. A fragmentação é conseqüência das atividades humanas, e as áreas de vegetação natural que restaram encontram-se geralmente próximas de áreas com perturbação antrópica, como fazendas agrícolas e de exploração florestal, estando sujeitas a tensão excessiva de agentes externos como fogo, inseticidas e espécie invasoras (JANSEN, 1986).

Fragmentos florestais localizados em área de intensa ação antrópica assemelham-se às ilhas oceânicas, separadas dos continentes aos quais estiveram ligadas em outras épocas. Estas ilhas biogeográficas encontram-se em equilíbrio quando o número de espécies presentes representa um balanço entre imigração e extinção. A taxa de imigração é determinada pelo grau de isolamento da ilha: quanto mais isolada estiver a ilha, mais baixa será a taxa de imigração. A taxa de extinção está diretamente ligada ao tamanho da ilha, sendo que ilhas maiores hospedam mais espécies, tanto de animais como plantas, do que ilhas menores, e desta forma são menos vulneráveis à extinção (MacARTHUR \& WILSON, 1967).

A distância entre os fragmentos e a diminuição das áreas naturais, dificultam a dispersão e reduzem o tamanho das populações. O tipo de vizinhança, a redução da área dos fragmentos e a alta relação borda/interior, contribuem para o aumento da pressão dos predadores, competidores, parasitas e doenças (ROLSTAD, 1991).

$\mathrm{O}$ grau de isolamento de um fragmento florestal, que pode ser considerado como a média das distâncias até os fragmentos mais próximos, pode afetar o influxo de animais, pólen e sementes e, portanto, a diversidade biológica e a dinâmica das populações (FORMAN \& GODRON, 1986).

Fragmentos isolados há muito tempo degeneram pela perda de animais polinizadores, dispersores e predadores, causando um desequilíbrio da flora e fauna (WHITMORE, 1991).

$\mathrm{O}$ aumento da complexidade estrutural da vegetação em vários níveis verticais possibilita novas formas de exploração do ambiente, e o aumento do número de espécies de aves se dá principalmente pelo aparecimento de novas guildas alimentares e pelo aumento no número de espécies das guildas já existentes (WILLSON, 1974).

O principal objetivo deste trabalho foi analisar os grupos de aves afetados pela fragmentação florestal numa região da Mata Atlântica e avaliar os impactos causados pela fragmentação e isolamento destas áreas.

\section{MATERIAL E MÉTODOS}

O estudo foi realizado na fazenda Cachoeira, de propriedade da Cia. Suzano de Papel e Celulose, localizada no Município de Paraibuna, Estado de São Paulo, Brasil, entre as coordenadas geográficas $23^{\circ} 31^{\prime}$ e $23^{\circ} 33^{\prime}$ de latitude Sul e $45^{\circ} 39^{\prime}$ e $45^{\circ} 42^{\prime}$ de longitude Oeste. A região enquadra-se, segundo a classificação de Köppen, no tipo climático Cfa, com precipitação atmosférica entre 1.400 e $1.500 \mathrm{~mm}$ anuais. Neste tipo climático, a temperatura média do mês mais frio é inferior a $18^{\circ} \mathrm{C}$ e a do mês mais quente é superior a $22^{\circ} \mathrm{C}$, sendo o total de chuvas no mês mais seco (agosto) superior a 30mm (VEIGA, 1985).

A fazenda está inserida na Serra do Mar, no domínio da Mata Atlântica, na formação florestal denominada Floresta Ombrófila Densa Atlântica, na subdivisão Montana, com altitude variando de 944 a $1.136 \mathrm{~m}$ do nível do mar.

Os ambientes estudados foram três fragmentos de vegetação natural identificados como $\mathrm{F}_{1}, \mathrm{~F}_{2}$ e $\mathrm{F}_{3}$, com respectivamente 47,26 e 4 hectares, circundados por plantios de eucaliptos em diferentes idades. Também foi estudada uma área de 40 hectares de plantio de eucaliptos com 18 anos de idade, adjacente aos fragmentos e com sub-bosque bem desenvolvido.

A vegetação dos fragmentos é uma mata ciliar em estádio médio de regeneração secundária, apresentando as mesmas características fisionômicas e composição florística bastante semelhante, com fisionomia arbórea e arbustiva predominando sobre a herbácea, constituindo estratos diferenciados. 
O estrato superior é formado por árvores emergentes distribuídas esparsadamente, que atingem até $25 \mathrm{~m}$ de altura, sendo as espécies com maior densidade: Aspidosperma parvifolium, Copaifera langsdorffii, Ocotea pulchella, Anadenanthera colubrina e Parapiptadenia rigida. Não há a formação de um dossel contínuo, o que garante uma penetração de luz suficiente que, juntamente com o elevado teor de umidade, favorece o desenvolvimento de musgos, liquens, epífitas e cipós no interior dos fragmentos.

O subdossel é formado por árvores com alturas entre 10 e $15 \mathrm{~m}$, com distribuição diamétrica apresentando amplitude moderada, com o predomínio de pequenos diâmetros (6 a $10 \mathrm{~cm})$, cobertura variando de aberta a fechada e grande diversidade de espécies, como Guatteria nigrescens, Tabebuia umbellata, Anadenanthera colubrina, Cordia superba, Alchornea triplinervia, Croton floribundus, Mabea fistulifera, Machaerium villosum, Casearia sylvestris, Nectandra lanceolata, Cedrela fissilis, Mimosa scabrella, Parapiptadenia rigida, Piptadenia gonoacantha e Campomanesia guazumifolia.

O sub-bosque é formado por indivíduos jovens das espécies arbóreas do dossel e subdossel, e arbustos principalmente das famílias Rubiaceae, Myrtaceae, Euphorbiaceae e Melastomataceae, atingindo até $4 \mathrm{~m}$ de altura. O estrato herbáceo é formado por pequenos arbustos, ervas e grande quantidade de bromélias dos gêneros Aechmea e Billbergia, principalmente nas áreas onde há maior incidência de luz. O solo é coberto por uma boa camada de serapilheira, com cerca de $10 \mathrm{~cm}$ de espessura.

O plantio de eucaliptos estudado compreende uma área de 40 hectares, formado pela espécie Eucalyptus smith, com 18 anos de idade. O espaçamento original era de $3 \times 2 \mathrm{~m}$, com árvores atingindo 25 a $30 \mathrm{~m}$ de altura e formando um dossel contínuo. O sub-bosque é denso e formado por arbustos e árvores jovens de até $5 \mathrm{~m}$ de altura.

O método utilizado para o registro da avifauna foi o de observações por pontos fixos, desenvolvido por Ferry \& Frochot (ROBBINS, 1978). Os pontos foram marcados com estacas numeradas, distantes cerca de $200 \mathrm{~m}$ entre si e distribuídas aleatoriamente ao longo das trilhas abertas no interior dos fragmentos e do plantio de eucaliptos, estabelecendo-se uma rede de pontos nos ambientes estudados, de modo a cobrir uma amostra representativa da comunidade a ser analisada. Foram realizados 20 minutos diários de observação em cada ponto, registrando-se em fichas todos os indivíduos que podiam ser identificados com segurança, anotando-se a espécie, quantidade, data, horário, local e estrato arbóreo, segundo FULLER \& LANGSLOW (1984). A distância de 200m entre pontos e a permanência de 20 minutos em cada ponto são adequadas para que não haja sobreposição de território de algumas espécies, e também por permitir uma boa amostragem da avifauna, segundo VIELLIARD \& SILVA (1989).

As observações foram realizadas durante as três primeiras horas após o amanhecer e antes do anoitecer, em seis períodos de amostragens, nas quatro estações do ano de 1997, totalizando 80 horas efetivas, distribuídas em 240 amostras. A identificação das aves foi visual, com o auxílio de binóculos e guias de campo, e auditiva (cantos, pios, chamadas e gritos de alerta), com o auxílio de gravador microcassete, sendo as gravações posteriormente identificadas em laboratório. Para evitar a sobreposição de território, considerando-se a distância mínima adotada de $200 \mathrm{~m}$, dividiu-se cada ponto em quatro quadrantes, orientando-se com uma bússola e anotando-se os indivíduos observados de acordo com a sua localização dentro do quadrante, evitando-se desta forma, registrar o mesmo indivíduo duas ou mais vezes. Em função da maior abundância de aves, não houve obrigatoriedade na utilização seqüencial dos pontos, aumentando-se neste caso, a distância entre os mesmos.

Houve um acompanhamento da estabilização das curvas do número acumulado de espécies, que coincidiu com o período final do levantamento, o que nos permite concluir que as horas utilizadas foram suficientes para o registro da maior parte das espécies da avifauna existentes.

O material bibliográfico utilizado na identificação da avifauna foi baseado nas publicações de SCHAUENSEE \& PHELPS Jr. (1978), FRISCH (1981), DUNNING (1985), GRANTSAU (1989) e SICK (1997). A nomenclatura científica e ordem taxonômica seguem a disposição proposta por SCHAUENSEE (1966).

A amostragem por pontos fixos resultou num levantamento quantitativo da avifauna das áreas estudadas, possibilitando obter o Índice Pontual de Abundância (IPA) para cada espécie registrada. O IPA indica a abundância de cada espécie em função do seu coeficiente de conspicuidade, através do número de contatos de determinada espécie e o número total de amostras.

A partir dos resultados obtidos, as aves foram agrupadas de acordo com a guilda alimentar, sendo consideradas oito guildas: frugívora, granívora, insetívora, inseto-carnívora, necrófaga, néctar-insetívora, onívora e piscívora. 
A ocupação vertical das áreas foi representada pela distribuição da avifauna de acordo com o nível predominante de cada espécie, baseado nos levantamentos de campo. Para tanto, foram considerados quatro níveis: aéreo, dossel, subbosque e solo.

\section{RESULTADOS E DISCUSSÃO}

Considerando-se todas as áreas estudadas, foi possível registrar 113 espécies de aves, distribuídas em 27 famílias e 13 ordens, sendo realizado 3.436 contatos. Considerou-se contato o indivíduo registrado no dia do levantamento, podendo ter havido vários contatos com o mesmo indivíduo, desde que em dias diferentes. $\mathrm{O}$ índice de densidade para os fragmentos F1, F2 e F3 foi respectivamente 56, 40 e 48 indivíduos contatados a cada hora de observação e 18 para o plantio de eucaliptos.

A diferença na quantidade de espécies entre os fragmentos florestais e o plantio de eucaliptos se deve, segundo MacARTHUR (1972), ao fato de a floresta proporcionar uma grande quantidade de nichos que abrigam uma diversidade de habitantes nos diferentes níveis da estratificação, tendo efeito direto na diversidade de aves. Esta diversidade de aves, segundo LEBRETON \& CHOISY (1991) está ligada mais diretamente à estrutura da floresta do que à quantidade de espécies vegetais existentes neste ambiente natural.

TOLEDO (1993), estudando a avifauna de duas reservas da Mata Atlântica localizadas na Serra da Mantiqueira, na região do Vale do Paraíba, Estado de São Paulo, registrou 102 espécies de aves distribuídas em 30 famílias. ALLEGRINI (1997), estudando a avifauna em diferentes estágios de regeneração da Mata Atlântica, nas encostas da Serra de Paranapiacaba, sul do Estado de São Paulo, registrou 217 espécies de aves distribuídas em 40 famílias, sendo que o número de espécies registradas em cada estágio foi 67 no pioneiro, 97 no inicial, 99 no médio e 79 no avançado.

Através do Índice Pontual de Abundância (IPA) obteve-se uma densidade estimada das espécies registradas. O IPA variou para o conjunto das áreas, de 0,0042 a 1,4917, ou seja, de 1 a 358 registros, em 80 horas de observação (Tabela 1), sendo as espécies de maior abundância Basileuterus leucoblepharus, Basileuterus culicivorus, Chiroxiphia caudata, Thamnophilus caerulescens, Platyrinchus mystaceus, Syndactyla rufosuperciliata, Sittasomus griseicapillus, Todirostrum plumbeiceps, Cyclarhis gujanensis, Conopophaga lineata. Estas espécies ocupam predominantemente o sub-bosque da floresta, e com exceção da Chiroxiphia caudata (onívora), todas pertencem à guilda insetívora.

Das 113 espécies observadas, 19 possuem IPA maior que 0,2500 , ou seja, foram registradas no mínimo 60 vezes em 80 horas de observação, 30 possuem IPA entre 0,0625 e 0,2458 (entre 15 e 59 registros), 50 tiveram de 2 a 14 registros e 14 apenas 1 registro, sendo que as 51 espécies de maior IPA foram registradas nos três fragmentos. $\mathrm{O}$ Índice de Similaridade de Jaccard foi $67,3 \%$ entre os dois maiores fragmentos $\left(\mathrm{F}_{1}\right.$ e $\left.\mathrm{F}_{2}\right) ; 65,7 \%$ entre o maior $\left(\mathrm{F}_{1}\right)$ e o menor fragmento $\left(\mathrm{F}_{3}\right) ; 66,3 \%$ entre os dois menores fragmentos $\left(\mathrm{F}_{2}\right.$ e $\left.\mathrm{F}_{3}\right) ; 41,9 \%$ entre o plantio de eucaliptos e o maior fragmento $\left(\mathrm{F}_{1}\right) ; 44,8 \%$ entre o plantio de eucaliptos e o fragmento intermediário $\left(\mathrm{F}_{2}\right) ; 46,5 \%$ entre o plantio de eucaliptos e o menor fragmento $\left(\mathrm{F}_{3}\right)$, o que indica haver semelhança entre os três fragmentos, quanto à avifauna presente. Nota-se que o plantio de eucaliptos possui maior similaridade com o menor fragmento $\left(\mathrm{F}_{3}\right)$, que está sofrendo maior efeito de borda.

Algumas espécies pouco abundantes nos dois fragmentos de maior área $\left(\mathrm{F}_{1}\right.$ e $\left.\mathrm{F}_{2}\right)$ têm grande destaque no fragmento de menor área $\left(\mathrm{F}_{3}\right)$, como o Tangara desmaresti, com o $3^{\circ}$ maior IPA, Sporophila caerulescens com o $5^{-}$, Thraupis sayaca com o $6^{\circ} \mathrm{e}$ Zonotrichia capensis com o $10^{\circ}$. O fato de as espécies onívoras e granívoras de sub-bosque terem maior densidade no $\mathrm{F}_{3}$ é devido ao maior efeito de borda que este ambiente está sofrendo, pois possui estreita faixa de vegetação natural. A maior incidência de luz neste ambiente proporciona maior produção de frutos e de plantas invasoras produtoras de sementes, que são a base alimentar destas espécies.

No plantio de eucaliptos destacam-se as espécies que possuem boa adaptação aos ambientes antropisados, como o Tyrannus melancholicus com o $5^{\mathrm{O}}$ maior IPA, Pitangus sulphuratus com o $8^{\mathrm{o}} \mathrm{e}$ Sporophila caerulescens com o $10^{\circ}$. No entanto, espécies tipicamente florestais e que tiveram alta freqüência nos fragmentos, não foram observadas no plantio de eucaliptos, como o Crypturellus obsoletus, Mackenziana severa, Dysithamnus mentalis, Drymophila malura, Drymophila squamata, Phylloscartes paulistus, Todirostrum cinereum, Myiobius barbatus, Myiophobus fasciatus, Attila rufus, Myiarchus ferox, Ilicura militaris, Geothlypis aequinoctialis e Tachyphonus coronatus. Tal fato demonstra que o plantio de eucaliptos, mesmo com o sub-bosque bem desenvolvido, constitui-se numa barreira para as espécies da avifauna, principalmente as florestais. Das 113 espécies observadas, 71 não foram registradas no plantio de eucaliptos. 
Tabela 1 - Índice pontual de abundância (IPA) para as espécies registradas em cada área estudada $\left(\mathrm{F}_{1}=\right.$ fragmento $1 ; \mathrm{F}_{2}=$ fragmento $2 ; \mathrm{F}_{3}=$ fragmento $3 ; \mathrm{E}=$ plantio de eucaliptos).

\begin{tabular}{|c|c|c|c|c|c|}
\hline Espécie & F1 & F2 & F3 & $\mathbf{E}$ & total \\
\hline Crypturellus obsoletus & 0,4154 & 0,3000 & 0,1846 & - & 0,2500 \\
\hline Coragyps atratus & 0,0308 & - & - & - & 0,0083 \\
\hline Buteo albicaudatus & - & 0,0143 & - & - & 0,0042 \\
\hline Buteo brachyurus & 0,0154 & - & - & - & 0,0042 \\
\hline Rupornis magnirostris & 0,1231 & 0,1286 & 0,0769 & 0,0500 & 0,1000 \\
\hline Herpetotheres cachinnans & - & 0,0143 & - & - & 0,0042 \\
\hline Micrastur ruficollis & - & 0,0143 & - & - & 0,0042 \\
\hline Milvago chimachima & 0,0769 & 0,0857 & 0,0923 & 0,1000 & 0,0875 \\
\hline Polyborus plancus & 0,0154 & 0,0286 & - & - & 0,0125 \\
\hline Penelope superciliaris & 0,0154 & - & - & - & 0,0042 \\
\hline Rallus nigricans & 0,0154 & 0,0143 & 0,0615 & - & 0,0250 \\
\hline Cariama cristata & 0,0154 & - & - & - & 0,0042 \\
\hline Columba picazuro & - & - & 0,0615 & 0,1000 & 0,0333 \\
\hline Columbina talpacoti & 0,0615 & 0,0857 & 0,1846 & 0,1000 & 0,1083 \\
\hline Leptotila verreauxi & 0,0154 & 0,1143 & 0,0308 & 0,0500 & 0,0542 \\
\hline Pionus maximiliani & 0,0154 & 0,0571 & - & - & 0,0208 \\
\hline Piaya cayana & 0,1231 & 0,0571 & 0,0615 & 0,0500 & 0,0750 \\
\hline Guira guira & - & 0,0571 & - & - & 0,0167 \\
\hline Nyctibius griseus & - & - & 0,0308 & - & 0,0083 \\
\hline Nyctidromus albicollis & 0,0308 & - & 0,0462 & - & 0,0208 \\
\hline Phaethornis eurynome & 0,2769 & 0,2571 & 0,4462 & 0,1500 & 0,2958 \\
\hline Phaethornis pretrei & 0,0462 & 0,0143 & 0,0615 & - & 0,0333 \\
\hline Melanotrochilus fuscus & 0,0308 & - & 0,0462 & - & 0,0208 \\
\hline Stephanoxis lalandi & - & 0,0143 & 0,0154 & - & 0,0083 \\
\hline Thalurania glaucopis & 0,0308 & - & 0,0308 & - & 0,0167 \\
\hline Leucochloris albicollis & 0,0923 & 0,0286 & 0,0769 & - & 0,0542 \\
\hline Amazilia fimbriata & 0,0154 & 0,0143 & 0,0154 & - & 0,0125 \\
\hline Aphantochroa cirrhochloris & - & 0,0143 & - & - & 0,0042 \\
\hline Chloroceryle americana & 0,0615 & 0,1143 & 0,1385 & - & 0,0875 \\
\hline Picumnus cirratus & 0,0615 & 0,0857 & 0,0769 & 0,0500 & 0,0708 \\
\hline Colaptes campestris & - & 0,0143 & 0,0462 & 0,0500 & 0,0250 \\
\hline Colaptes melanochloros & 0,0154 & - & - & - & 0,0042 \\
\hline Veniliornis spilogaster & 0,0308 & - & 0,0462 & 0,0250 & 0,0250 \\
\hline Mackenziaena severa & 0,2308 & 0,2143 & 0,1846 & - & 0,1750 \\
\hline Thamnophilus doliatus & - & 0,0143 & 0,1077 & 0,0750 & 0,0458 \\
\hline Thamnophilus caerulescens & 0,8923 & 0,7143 & 0,4462 & 0,2250 & 0,6083 \\
\hline Dysithamnus mentalis & 0,1538 & 0,0429 & 0,0769 & - & 0,0750 \\
\hline Drymophila malura & 0,1231 & 0,1286 & 0,0923 & - & 0,0958 \\
\hline Drymophila squamata & 0,0615 & 0,0429 & 0,1385 & - & 0,0667 \\
\hline Pyriglena leucoptera & 0,3077 & 0,2000 & 0,2923 & 0,1250 & 0,2417 \\
\hline Conopophaga lineata & 0,4923 & 0,3286 & 0,3846 & 0,1750 & 0,3625 \\
\hline Synallaxis spixi & 0,2615 & 0,0857 & 0,1231 & 0,0500 & 0,1375 \\
\hline Synallaxis ruficapilla & 0,4000 & 0,3429 & 0,2769 & 0,1500 & 0,3083 \\
\hline Phacellodomus rufifrons & 0,0615 & - & - & - & 0,0167 \\
\hline Phacellodomus ferrugineigula & 0,0462 & 0,1000 & 0,1231 & 0,0500 & 0,0833 \\
\hline Syndactyla rufosuperciliata & 0,7077 & 0,3429 & 0,3692 & 0,2000 & 0,4250 \\
\hline Lochmias nematura & 0,2923 & 0,2714 & 0,3692 & 0,2250 & 0,2958 \\
\hline Sittasomus griseicapillus & 0,5846 & 0,4143 & 0,4308 & 0,1000 & 0,4125 \\
\hline Lepidocolaptes fuscus & 0,2308 & 0,0857 & 0,0769 & 0,0750 & 0,1208 \\
\hline Camptostoma obsoletum & 0,0923 & 0,0286 & - & - & 0,0333 \\
\hline Elaenia flavogaster & - & 0,0286 & 0,1846 & - & 0,0583 \\
\hline Elaenia parvirostris & 0,0154 & 0,0714 & 0,2154 & 0,0750 & 0,0958 \\
\hline Elaenia cristata & - & - & 0,0615 & - & 0,0167 \\
\hline Elaenia obscura & - & 0,0143 & - & - & 0,0042 \\
\hline Serpophaga subcristata & - & 0,0143 & - & - & 0,0042 \\
\hline Mionectes rufiventris & 0,0769 & 0,0286 & - & - & 0,0292 \\
\hline Leptopogon amaurocephalus & 0,0154 & 0,0286 & - & - & 0,0125 \\
\hline Phylloscartes paulistus & 0,0154 & 0,0857 & 0,0308 & - & 0,0375 \\
\hline
\end{tabular}

Das 113 espécies registradas, 61 (54\%) são comuns aos três fragmentos. Destas 61 espécies, 36 são as que possuem as maiores densidades populacionais no $\mathrm{F}_{1}, 47$ possuem as maiores densidades no $\mathrm{F}_{2}$ e 31 possuem as maiores densidades no $\mathrm{F}_{3}$. Foram registradas 90 espécies no $F_{1}, 84$ no $F_{2}, 84$ no $F_{3}$ e 42 no plantio de eucaliptos, sendo 10 espécies exclusivas do $F_{1}, 8$ exclusivas do $F_{2}$ e 9 exclusivas do $F_{3}$, não havendo nenhuma espécie exclusiva do plantio de eucaliptos.

Para todas as áreas estudadas, há uma predominância de indivíduos pertencentes à guilda insetívora que ocupam o sub-bosque da floresta, seguindo-se os onívoros e granívoros que também 


\begin{tabular}{|c|c|c|c|c|c|}
\hline Espécie & F1 & $\mathbf{F} 2$ & F3 & $\mathbf{E}$ & total \\
\hline \multicolumn{6}{|l|}{ Continuação... } \\
\hline Capsiempis flaveola & 0,0308 & 0,0143 & 0,0615 & - & 0,0292 \\
\hline Myiornis auricularis & 0,0154 & - & - & - & 0,0042 \\
\hline Todirostrum poliocephalum & - & 0,0143 & 0,0308 & - & 0,0125 \\
\hline Todirostrum cinereum & 0,1538 & 0,0286 & - & - & 0,0500 \\
\hline Todirostrum plumbeiceps & 0,7077 & 0,2143 & 0,3538 & 0,1500 & 0,3750 \\
\hline Tolmomyias sulphurescens & 0,4615 & 0,2429 & 0,2615 & 0,1250 & 0,2875 \\
\hline Platyrinchus mystaceus & 0,2769 & 0,6571 & 0,6615 & 0,3750 & 0,5083 \\
\hline Myiobius barbatus & 0,0615 & 0,0429 & 0,1231 & - & 0,0625 \\
\hline Myiophobus fasciatus & 0,1077 & 0,0286 & 0,1231 & - & 0,0708 \\
\hline Lathrotriccus euleri & 0,1692 & 0,2286 & 0,2000 & 0,1000 & 0,1833 \\
\hline Knipolegus lophotes & 0,0154 & - & - & - & 0,0042 \\
\hline Knipolegus cyanirostris & 0,0308 & 0,0143 & - & - & 0,0125 \\
\hline Attila rufus & 0,2462 & 0,0857 & 0,1385 & - & 0,1292 \\
\hline Myiarchus ferox & 0,1077 & 0,0143 & 0,0462 & - & 0,0458 \\
\hline Pitangus sulphuratus & 0,2769 & 0,1286 & 0,3538 & 0,2250 & 0,2458 \\
\hline Myiozetetes similis & 0,0308 & - & - & - & 0,0083 \\
\hline Empidonomus varius & 0,0615 & 0,0429 & - & 0,0250 & 0,0333 \\
\hline Tyrannus savana & - & - & 0,0615 & - & 0,0167 \\
\hline Tyrannus melancholicus & 0,0615 & 0,1143 & 0,3846 & 0,2750 & 0,2000 \\
\hline Pachyramphus polychopterus & 0,2615 & 0,0714 & 0,0308 & 0,0750 & 0,1125 \\
\hline Chiroxiphia caudata & 0,8769 & 0,9429 & 0,4000 & 0,3750 & 0,6833 \\
\hline Ilicura militaris & 0,0615 & 0,0714 & 0,0923 & - & 0,0625 \\
\hline Cyanocorax cristatellus & 0,0154 & - & - & - & 0,0042 \\
\hline Troglodytes aedon & 0,0462 & - & 0,0308 & - & 0,0208 \\
\hline Platycichla flavipes & 0,0308 & 0,0143 & 0,0462 & - & 0,0250 \\
\hline Turdus rufiventris & 0,3538 & 0,2286 & 0,3538 & 0,1500 & 0,2833 \\
\hline Turdus amaurochalinus & 0,1846 & 0,0571 & 0,1692 & 0,0500 & 0,1208 \\
\hline Turdus albicollis & 0,1077 & 0,0429 & 0,0615 & - & 0,0583 \\
\hline Cyclarhis gujanensis & 0,6462 & 0,3714 & 0,2462 & 0,1250 & 0,3708 \\
\hline Vireo olivaceus & 0,0923 & - & 0,0769 & - & 0,0458 \\
\hline Hylophilus poicilotis & 0,0462 & - & - & 0,0250 & 0,0167 \\
\hline Hylophilus thoracicus & - & 0,0286 & - & - & 0,0083 \\
\hline Parula pitiayumi & 0,4308 & 0,2000 & 0,1846 & 0,0500 & 0,2333 \\
\hline Geothlypis aequinoctialis & 0,1846 & 0,1000 & 0,1692 & - & 0,1250 \\
\hline Basileuterus culicivorus & 1,4462 & 1,3714 & 0,8154 & 0,4000 & 1,0792 \\
\hline Basileuterus leucoblepharus & 2,3538 & 1,4429 & 1,2000 & 0,6500 & 1,4917 \\
\hline Coereba flaveola & - & - & 0,0308 & - & 0,0083 \\
\hline Hemithraupis ruficapilla & 0,0615 & 0,0429 & 0,0615 & - & 0,0458 \\
\hline Tachyphonus coronatus & 0,2462 & 0,2571 & 0,3692 & - & 0,2417 \\
\hline Trichothraupis melanops & 0,1538 & 0,0714 & 0,2154 & 0,0750 & 0,1333 \\
\hline Thraupis sayaca & 0,1692 & 0,1571 & 0,5538 & - & 0,2417 \\
\hline Stephanophorus diadematus & - & 0,0286 & 0,0615 & - & 0,0250 \\
\hline Pipraeidea melanonota & - & - & 0,0923 & - & 0,0250 \\
\hline Euphonia chlorotica & 0,0154 & - & 0,0923 & - & 0,0292 \\
\hline Tangara desmaresti & 0,1846 & 0,0571 & 0,7077 & - & 0,2583 \\
\hline Tangara cayana & - & - & 0,0308 & - & 0,0083 \\
\hline Dacnis cayana & - & - & 0,0615 & - & 0,0167 \\
\hline Conirostrum speciosum & 0,1231 & 0,0286 & - & - & 0,0417 \\
\hline Zonotrichia capensis & 0,3077 & 0,1714 & 0,4308 & 0,1750 & 0,2792 \\
\hline Volatinia jacarina & 0,0308 & - & 0,1231 & - & 0,0417 \\
\hline Sporophila caerulescens & 0,2462 & 0,2000 & 0,6000 & 0,2000 & 0,3208 \\
\hline Arremon flavirostris & 0,0154 & - & - & - & 0,0042 \\
\hline Saltator similis & 0,4154 & 0,1857 & 0,2154 & 0,1000 & 0,2417 \\
\hline Carduelis magellanicus & - & - & 0,0308 & - & 0,0083 \\
\hline
\end{tabular}

ocupam o mesmo ambiente, mostrando a importância da existência do sub-bosque, principalmente nos plantios de eucaliptos, onde a diversidade vegetal geralmente é menor (Tabela 2).

Comparando-se os dados obtidos por WILLIS (1979), quanto às guildas ocupadas pelas espécies de aves da floresta de Barreiro Rico, no Município de Anhembi, Estado de São Paulo e de ALMEIDA (1982), em fragmentos de matas ciliares na mesma região, observa-se que, nas matas ciliares e capoeiras, predominam as espécies insetívoras, onívoras e granívoras que vivem na borda da floresta. Este fato é constatado nos fragmentos estudados, tipicamente constituídos por mata ciliar com vegetação em estádio médio de regeneração, onde $75 \%$ das espécies registradas pertencem à guilda insetívora, onívora ou granívora.

ALMEIDA (1982) constatou que as maiores reduções quanto à fragmentação de habitats ocorrem entre as espécies insetívoras, seguindo-se as onívoras de grande porte que se alimentam no chão e as onívoras que se alimentam de insetos e frutos grandes.

Dentre as espécies onívoras de grande porte que se alimentam no chão, houve o registro apenas do Crypturellus obsoletus, de grande valor cinegético e que habita matas densas. Segundo TERBORGH \& WESKE (1969), esta espécie está entre os representantes mais ameaçados de extinção pela fragmentação de áreas florestais. 
Tabela 2 - Porcentagem dos indivíduos de diferentes guildas observados em cada área estudada $\left(\mathrm{F}_{1}=\right.$ fragmento $1 ; \mathrm{F}_{2}=$ fragmento $2 ; \mathrm{F}_{3}=$ fragmento $3 ; \mathrm{E}=$ plantio de eucaliptos).

\begin{tabular}{lcccc}
\hline \multicolumn{1}{c}{ Guilda } & $\mathrm{F}_{1}$ & $\mathrm{~F}_{2}$ & $\mathrm{~F}_{3}$ & $\mathrm{E}$ \\
\hline Insetívora de sub-bosque & 64,59 & 63,96 & 52,61 & 57,32 \\
Onívora de sub-bosque & 17,21 & 17,86 & 22,22 & 12,97 \\
Granívora de sub-bosque & 5,82 & 5,73 & 9,97 & 10,46 \\
Insetívora de dossel & 3,93 & 2,71 & 5,51 & 10,46 \\
Néctar-insetívora de sub-bosque & 2,62 & 2,60 & 4,46 & 2,51 \\
Granívora de solo & 2,21 & 2,27 & 1,52 & 1,67 \\
Inseto-carnívora de dossel & 1,88 & 3,03 & 1,42 & 3,35 \\
Outras & 1,74 & 1,84 & 2,29 & 1,26 \\
\hline
\end{tabular}

Houve o registro de um número pequeno de espécies umbrófilas nas áreas estudadas, e uma grande densidade de espécies adaptadas aos ambientes abertos, como o Polyborus planchus, Buteo albicaudatus, Milvago chimachima, Cariama cristata e Colaptes campestris, espécies típicas de ambientes abertos, e que foram observadas principalmente na borda dos fragmentos. Segundo HÖFLING \& LENCIONI (1992), a fauna nesta região é predominantemente umbrófila, pouco tolerante às variações de umidade relativa e da temperatura, assim como insolação elevada. A destruição e fragmentação das áreas florestais teriam o efeito imediato de eliminar das áreas afetadas praticamente toda a fauna umbrófila. A redução das áreas de florestas naturais, assim como o efeito de borda sobre os fragmentos florestais não afetam apenas a fauna das áreas perturbadas, mas também das áreas limítrofes à perturbação.

\section{CONCLUSÕES}

A redução e fragmentação dos ambientes naturais afetaram a dinâmica das populações da avifauna existentes, diminuindo o número de espécies e eliminando praticamente toda a avifauna umbrófila, que é pouco tolerante às variações de temperatura e umidade.

A diversidade da vegetação e o número de estratos definidos estão diretamente ligados à diversidade e densidade da avifauna, assim como o tamanho do fragmento e o grau de isolamento, sendo que a fragmentação dos ambientes estudados parece que afetou principalmente as espécies raras e de baixa densidade populacional.

O efeito de borda sobre os ambientes favorecem as espécies de aves onívoras e granívoras que habitam o sub-bosque da floresta. A maior incidência de luz nestes ambientes proporciona maior produção de frutos e de plantas invasoras produtoras de sementes, que são a base alimentar destas espécies.

\section{REFERÊNCIAS BIBLIOGRÁFICAS}

ALLEGRINI, M.F. Avifauna como possível indicador biológico dos estádios de regeneração da Mata Atlântica. São Paulo, SP, 1997. 161p. Dissertação (Mestrado em Ciência Ambiental) - Universidade de São Paulo, 1997.

ALMEIDA, A.F. Análise das categorias de nichos tróficos das aves de matas ciliares em Anhembi, Estado de São Paulo. In: CONGRESSO NACIONAL SOBRE ESSÊNCIAS NATIVAS, 1982, Campos do Jordão. Anais... São Paulo : Instituto Florestal, 1982. V.3, p.1787-1795.

DÁRIO, F.R., ALMEIDA, A.F. Influência de corredor florestal sobre a avifauna da Mata Atlântica. Scientia Forestalis, n.58, p.99-109, 2000.

DUNNING, J.T. South american birds: a photographic aid to identification. Pennsylvania : Newtown Square, 1985. 122p.

FORMAN, R.T.T., GODRON, M. Landscape ecology. New York : John Wiley, 1986. 619p.

FRISCH, J.D. Aves brasileiras. São Paulo : Dalgas Ecoltec, 1981. 353p.

FULLER, R.J., LANGSLOW, D.R. Estimating numbers of birds by point counts: how long should counts last? Bird Study, v.31, p.195-202, 1984

GRANTSAU, R. Os beija-flores do Brasil. Rio de Janeiro: Expressão e Cultura, 1989. 233p.

HARRIS, L.D. The fragmented forest: island biogeography theory and the preservation of biotic diversity. Chicago: University of Chicago, 1984. 229p.

HÖFLING, E., LENCIONI, F. Avifauna da floresta atlântica, região de Salesópolis, Estado de São Paulo. Revista Brasileira de Biologia, v.52, p.361-378, 1992.

JANSEN, D.H. The eternal external threat. In: SOULÉ, M.E. Conservation biology: the science of scarcity and diversity. Sinauer Associates, 1986. p.286-303.

JOLY, C.A., LEITÃO FILHO; H.F., SILVA, S.M. O patrimônio florístico. In: CAMARA, I.G. Mata atlântica. São Paulo : Fundação SOS Mata Atlântica, 1991. p.62-89.

LEBRETON, P., CHOISY, J.P. Incidences avifaunistiques des amenagements forestieres: substitutions Quercus/Pinus en milieu submediterraneen. Bulletin d'Ecologie. n.22, p.213220, 1991.

LEITÃo FILHO, H.F. Aspectos taxonômicos das florestas do Estado de São Paulo. In: CONGRESSO NACIONAL SOBRE ESSÊNCIAS NATIVAS, 1982, Campos do Jordão, SP. Anais... São Paulo : Instituto Florestal, 1982. p.197-206.

MacARTHUR, R.H. Geographical ecology: patterns in the distribution of species. New York : Harper \& Row, 1972. 269 p.

MacARTHUR, R.H.; WILSON, E.O. The theory of island biogeography. Princeton: Princeton University, 1967. 203p. 
MacARTHUR, R.H., WHITMORE, R.C. Passerine community composition and diversity in man-altered environments. Morgantown, West Virginia, v.7, p.1-12, 1979.

ROBBINS, C.S. Census techniques for forest birds. In: WORKSHOP MANAGEMENT OF SOUTHERN FORESTS FOR NONGAME BIRDS, 1978, Atlanta, USA. Anais.... Atlanta : Forest Service, 1978. V.14, p.142-163.

ROLSTAD, J. Consequences of forest fragmentation for the dynamics of bird populations: conceptual issues and the evidence. Biological Journal of the Linnean Society, v.42, p.149-163, 1991.

SCHAUENSEE, R.M. The species of birds of South America and their distribution. Narberth : Livingston, 1966. $88 \mathrm{p}$

SCHAUENSEE, R.M., PHELPS Jr, W.R. Una guia de las aves de Venezuela. Princeton : Princeton University, 1978. $484 p$.

SICK, H. Ornitologia brasileira. Rio de Janeiro : Nova Fronteira, 1997. 862p.
TERBORGH, J., WESKE, J.S. Colonization of secondary habitats by Peruvian birds. Ecolgy monographs, Durham, v.50, p.765-782, 1969.

TOLEDO, M.C.B. Avifauna em duas reservas fragmentadas de Mata Atlântica na Serra da Mantiqueira, São Paulo. Piracicaba, SP, 1993. 112p. Dissertação (Mestrado em Ciências) - Universidade de São Paulo, 1993.

VEIGA, A.A. Coletânea de assuntos técnicos: área de dasonomia. São Paulo : Instituto Florestal, 1985. 139p.

VIELLIARD, J.M.E., SILVA, W.R. Nova metodologia de levantamento quantitativo da avifauna e primeiros resultados no interior do Estado de São Paulo, Brasil. In: ENAV, 4. Recife, 1989. Anais... Recife : UFRPe, 1989. p.117-151.

WHITMORE, T.C. An introduction to tropical rain forest. Clarendo : Oxford, 1991. 226p.

WILLIS, E.O. The composition of avian communities in remanescent woodlots in southern Brazil. Papéis Avulsos de Zoologia, São Paulo, v.33, p.1-25, 1979.

WILLSON, M.F. Avian community organization and habitat structure. Ecology monographs, v.55, p.1017-1029, 1974 